between a referral being made and the assessment taking placed varied between 1.5 hours and 22 hours. Two defendants were remanded overnight in prison as the MHAA could not take place on the same day as the referral.

In the 25 cases where an application for detention under Section 2 of the MHA was made, beds were not available on the same day in 7 cases. In 4 cases defendants required remand in prison custody due to beds not being available.

Conclusion. There were some limitations to this audit as data were not available for all 42 individuals referred for a MHAA.

Individuals referred for MHAA by the Service had both medical recommendations completed within 5 days and those who required admission to hospital were admitted within 14 days of the recommendations being completed.

Whilst these standards are being met, individuals referred for MHAA and those requiring admission to hospital are still facing remand to custody.

\section{Handcuff use on forensic psychiatry wards}

Rachel Swain*, Deborah Klayman, David Reiss, Kruti Buch and Sarah Roberts

West London NHS Trust

${ }^{\star}$ Corresponding author.

doi: 10.1192/bjo.2021.934

Aims. This project aimed to assess the use of handcuffs in a secure forensic mental health hospital.

Background. Handcuffs are used by secure forensic psychiatric wards where patients need to leave the ward and require added restrictive measures for their own or other's safety. The decision to use handcuffs is made by the multi-disciplinary team, with the input of the unit's clinical security team and is assessed based on individual risk and need. This study investigated the frequency, duration and purpose of handcuff use in one secure forensic mental health unit, encompassing 8 male medium secure wards, 5 male low secure wards, 1 adolescent secure ward, 1 female low secure ward and 5 female medium secure wards.

Method. Handcuff use was recorded contemporaneously by ward staff in a specialised handcuff proforma. This data were then compiled to assess the number of instances of use, the mental health section applicable to the patient, the reason the patient needed to leave the unit, and the duration of use (including the time period for which the handcuffs were removed during the visit, if applicable.) Data from these forms over an 18 month period were analysed.

Result. Over the 18 months, there were a total of 347 uses of handcuffs, with an average of 18.3 occurrences per month. In 55 cases, the patients were detained under a civil section, with the remaining instances occurring in patients detained under forensic section. $47 \%$ were unsentenced prisoners.

The most common destination for patients was the general medical hospital, which accounted for $49 \%$ of all visits. Court was the second most common destination, with $39 \%$ of uses.

The average duration spent in handcuffs was 3.3 hours. The average time that the handcuffs were taken off during the transfer was 1.2 hours.

Conclusion. Through ongoing education and supervision by the clinical security team, handcuff use in this forensic service was limited to essential situations, most often to allow treatment of physical health issues off-site. A large proportion of instances involved unsentenced prisoners and court attendances, where the risk of absconsion might be particularly high. Duration spent in handcuffs was kept to a minimum, with cuffs being removed where possible. The service strives to continue such good practices and to identify further ways to reduce handcuff use, such as using videoconferencing as an alternative court attendance.

\section{Assessment and management of patients detained under Section 136 in Northwick Park Hospital emergency department}

\author{
Laurence Telesia ${ }^{1 \star}$ and Lauren Fraser ${ }^{2}$ \\ ${ }^{1}$ South London and Maudsley NHS Foundation Trust and \\ ${ }^{2}$ Northwick Park Hospital, London North West University \\ Healthcare NHS Trust \\ ${ }^{\star}$ Corresponding author.
}

doi: 10.1192/bjo.2021.935

Aims. To evaluate the role of the Emergency Medicine team (EM) within a London Emergency Department (ED) in assessing and managing patients detained under Section 136 of the Mental Health Act, 1983 (S136).

Background. S136 allows detention and transfer of people to ED and psychiatric hospitals for further assessment. EDs are optimised for the investigation and management of the medically unwell, but attending ED may also delay access to psychiatric services if required. Minimal research has been performed to investigate the relative benefits of transferring people under S136 to ED versus psychiatric hospitals.

Method. Electronic notes were searched to identify those attending under S136 between 01/04/2017 and 31/03/2018. Scanned medical notes were reviewed and data extracted regarding patient demographics, length of ED stay, reason for S136 use, investigations and interventions undertaken by EM.

Result. This identified 95 attendances by 87 patients. The mean age was 35 years (range 15-75) and 59\% of attenders were male. The mean duration of stay was 7 hours 34 minutes (range 6 minutes - 25 hours 50 minutes).

Reasons for S136 use were abnormal behaviour (32), expressed suicidal ideation (29), overdose (15), self-harm (13), overdose plus self-harm (4), being found wandering (1) and was unclear for 1 presentation.

In 39 attendances no investigations beyond history and examination were performed by EM. Only 6 patients had investigations that were not bloods, electrocardiogram or urinalysis. These included X-radiograph trunk (4), computed tomography (CT) head (3), X-radiograph limb (3), CT cervical spine (1), Focused Assessment with Sonography for Trauma (1).

No interventions were given by EM in 55 attendances. Twenty-nine different medications were prescribed and 18 patients were prescribed intravenous fluids. Three had wounds dressed, 3 glued, 3 sutured and 1 stapled.

Conclusion. There were difficulties categorising the reason for S136 use, as clear documentation was often unavailable, but the vast majority of patients were detained due to abnormal behaviour, expressed suicidal ideation and self-harm.

Few attending ED under S136 received investigations or interventions that could not be offered within a psychiatric hospital. There was a wide range in duration of stay within ED, however $65 \%$ of attendances were longer than the standard 4 hour target.

Future research may assess the relative benefits of ED versus psychiatric hospitals in assessing those detained. This could aid services in meeting both the physical and psychiatric needs of patients whilst making efficient use of available resources. 